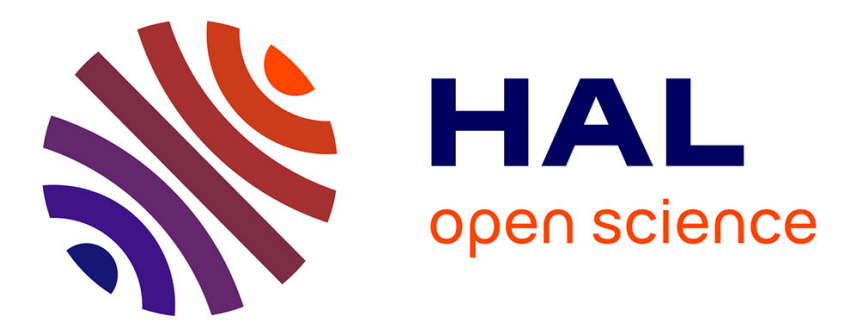

\title{
Super-elastic electron scattering from the laser-excited 41P1 state of calcium at low incident energy
}

Alex Knight-Percival, Sarah Jhumka, Martyn Hussey, Andrew James Murray

\section{To cite this version:}

Alex Knight-Percival, Sarah Jhumka, Martyn Hussey, Andrew James Murray. Super-elastic electron scattering from the laser-excited 41P1 state of calcium at low incident energy. Journal of Physics B: Atomic, Molecular and Optical Physics, 2011, 44 (10), pp.105203. 10.1088/0953-4075/44/10/105203 . hal-00617387

\section{HAL Id: hal-00617387 https://hal.science/hal-00617387}

Submitted on 28 Aug 2011

HAL is a multi-disciplinary open access archive for the deposit and dissemination of scientific research documents, whether they are published or not. The documents may come from teaching and research institutions in France or abroad, or from public or private research centers.
L'archive ouverte pluridisciplinaire HAL, est destinée au dépôt et à la diffusion de documents scientifiques de niveau recherche, publiés ou non, émanant des établissements d'enseignement et de recherche français ou étrangers, des laboratoires publics ou privés. 
Super-elastic electron scattering from the laser-excited $4^{1} \mathrm{P}_{1}$ state of Calcium at low incident energy.

Alex Knight-Percival, Sarah Jhumka, Martyn Hussey and Andrew James Murray* Photon Science Institute, School of Physics \& Astronomy

The University of Manchester, Manchester M13 PL, United Kingdom

*Email : Andrew.Murray@manchester.ac.uk

PACS No. 34.80

\begin{abstract}
.
Atomic Collision Parameters have been measured for electron impact excitation of calcium using the superelastic scattering method, at incident electron energies equivalent to $\sim 10 \mathrm{eV}$ and $\sim 12 \mathrm{eV}$. The parameters $P_{\text {lin }}, L_{\perp}$ and $\gamma$ were derived for the $4{ }^{1} \mathrm{P}_{1}$ state, and the related Stokes parameters determined. The results are compared to previously published calculations from four different theories: a relativistic distorted wave calculation, an R-matrix calculation, an R-matrix theory using B-splines, and a Convergent Close Coupling theory.
\end{abstract}

Keywords: Super-elastic scattering, calcium, Atomic Collision Parameters, Stokes parameters. 


\subsection{Introduction.}

Derivation of the Atomic Collision Parameters (ACP's) from electron excitation of atoms $[1,2]$ provides the most sensitive test of different scattering theories leading to excitation of atomic targets. In these experiments, an electron of incident energy $E_{i n c}^{c}$ collides with an atomic target so as to excite the atom to a state with internal energy $E_{P}$ (see figure 1a). The outgoing electron of energy $E_{\text {out }}^{c}=E_{\text {inc }}^{c}-E_{P}$ scatters from the target through an angle $\theta_{e}$. If the target is left in a P-state (as is usual in most experiments to date) the atomic charge cloud may be aligned at an angle $\gamma$ with respect to the incident electron trajectory, or the collision may impart angular momentum to the target. In the former case the alignment is described by a parameter $P_{\text {lin }}$, which determines the relative length of the charge cloud to the width. $\gamma$ then describes the direction of the major axis of the charge cloud, as shown in figure 1a. In the latter case, the angular momentum vector is orthogonal to the scattering plane, and has an expectation value given by the parameter $L_{\perp}$. A further parameter $\rho_{00}^{A}$ is also defined that relates to the possibility of spin-flip of the electron during the interaction.

The atomic collision parameters $\left(P_{l i n}, \gamma, L_{\perp}, \rho_{00}^{A}\right)$ can be determined in several ways. Coincidence techniques can be used to measure the polarization of photons emitted orthogonal to the scattering plane from the excited atom as it relaxes to a lower state, the photons being time-correlated to electrons that scatter from the excited atom. These measurements determine the polarization using Stokes parameters $\left(P_{1}, P_{2}, P_{3}\right)$, which form a complete set that fully describe the polarization of light. $P_{1}$ and $P_{2}$ reveal the linear components of the radiation, and can be directly related to $P_{\text {lin }}$ and $\gamma$ through the relationships:

$$
\left.\begin{array}{l}
P_{1}=P_{\text {lin }} \cos 2 \gamma \\
P_{2}=P_{\text {lin }} \sin 2 \gamma
\end{array}\right\}
$$

where both $P_{1}$ and $P_{2}$ are here determined with respect to the direction of the incident electron. $P_{3}$ is related to the parameter $L_{\perp}$ through the relationship:

$$
P_{3}=-L_{\perp}
$$


The parameter $\rho_{00}^{A}$ is determined by measuring the polarization $P_{4}$ of photons emitted in the scattering plane. In this case the relationship is more complex and is given by:

$$
P_{4}=\frac{1-3 \rho_{00}^{A}+P_{\text {lin }}\left(1-\rho_{00}^{A}\right) \cos 2 \gamma}{1+\rho_{00}^{A}+P_{\text {lin }}\left(1-\rho_{00}^{A}\right) \cos 2 \gamma}
$$

The coincidence technique is a well established method that allows the ACP's to be fully determined. However, a problem with this technique is low yield, due to the high probability that the photon correlated with the detected electron is emitted in a direction away from the photon detector. In this case no coincidence is observed. The low coincidence yield from these experiments has hence limited the technique to small scattering angles, where the count rates are relatively high.

A contrasting technique that also determines the ACP's is the super-elastic scattering method, pioneered by Hertel and co-workers $[3,4]$. This method uses time-reversal arguments to provide an equivalent set of measurements. In this case, the experiment starts with a photon from a laser beam of equal energy to that emitted by the atom in the coincidence measurement. This photon is set to have well defined polarization, and is directed in the opposite direction to that emitted in coincidence studies. The electron source (the gun) is moved to the position of the electron analyser, and is adjusted to produce electrons of energy $E_{\text {inc }}^{s}=E_{\text {out }}^{c}$. The electron analyser moves to the position of the source, and is set to detect electrons of energy $E_{\text {out }}^{s}=E_{\text {inc }}^{c}$. Since the detected electrons now have a larger energy than $E_{i n c}^{s}$, they can only arise from de-excitation of the laser-excited atom, and so are superelastically scattered. Figure $1 \mathrm{~b}$ shows the geometry adopted in these experiments.

The key advantage of the super-elastic method is that the laser beam is always directed orthogonal to the scattering plane, eliminating the restrictions suffered by the coincidence technique described above. Further, since time correlation techniques are no longer required, it is only necessary to measure the rate of super-elastically scattered electrons at a given scattering angle $\theta_{e}$ as a function of the polarization of the laser beam. The count rates hence are far higher than for coincidence studies, yielding better overall statistical accuracy. Since the rates are higher, it is also possible to determine the ACP's over a much larger range of scattering angles [5]. 
The super-elastic technique also has limitations, principally due to the restricted number of targets that can be excited by existing laser radiation. These targets are mainly limited to alkali and alkali-earth atoms [see for example, 5-15], although new methods are under development that will allow a much larger number of atoms to be excited. A further complexity arises due to the high intensity of the laser radiation, which leads to Rabi-cycling and optical pumping of the atoms under study. Optical pumping becomes significantly more complex for targets with hyperfine structure (such as alkali atoms), and so an extensive experimental and theoretical study has been undertaken to determine these effects [16-18]. It has been shown that the laser interaction leads to an optical pumping parameter that can be decoupled from the electron collision, allowing the ACP's to be determined from the superelastic measurements. For atoms with hyperfine structure, the uncertainty in the optical pumping parameter leads to an additional uncertainty in the derived ACP's. For atoms with no hyperfine structure (such as calcium), the ACP's can be directly related to the super-elastic measurements.

A number of different collision theories have been applied to the study of the ACP's. At relatively high incident energies, models such as the distorted wave Born approximation (DWBA) and their relativistic derivatives (RDW) have proven accurate in describing the interactions for a range of targets [19]. Other models with proven success are the convergent close coupling (CCC) methods [20] and R-matrix techniques [21,22]. For the calcium target under study here, the RDW model is known to be accurate at incident energies above $25 \mathrm{eV}$ [19], however agreement between theory and experiment was seen to significantly deteriorate below this energy. This is not surprising as the RDW model is expected to become less accurate as the energy is lowered. By contrast, CCC and R-matrix calculations show excellent agreement with experiment for all energies down to $20 \mathrm{eV}$ [20-22], and further predict results at energies as low as $10 \mathrm{eV}$. At $10 \mathrm{eV}$, differences are seen in the Stokes parameters predicted by the different models, and so the motivation of the experiments detailed here was to provide data to test these predictions.

Experimental results are hence presented for super-elastic scattering from calcium at incident energies $E_{\text {inc }}^{c} \sim 10 \mathrm{eV}$ and $\sim 12 \mathrm{eV}$. The parameters $P_{\text {lin }}, \gamma$ and $L_{\perp}$ were directly determined using laser radiation from a frequency doubled Ti:Sapphire laser operating at $\sim 423 \mathrm{~nm}$. $\rho_{00}^{A}$ was not measured. From these results the Stokes parameters are inferred using equations 1 
and 2, and the results compared to predictions from CCC [20], R-matrix [21], RDW [19] and B-Spline R-matrix [22] calculations at $10 \mathrm{eV}$.

This paper is hence divided into four sections. Following this introduction the apparatus is described, and the techniques used to obtain the experimental data and derived ACP's are detailed. The experimental results are then presented, and are compared to predictions from the different models at $10 \mathrm{eV}$. Conclusions and a summary of future work are then given.

\subsection{Experimental techniques.}

Figure 2 depicts the apparatus used in these experiments. An electron gun produces a beam of well defined momentum that passes through the interaction region created by an atomic beam and laser beam. The incident electron energy was set to $\sim 7.1 \mathrm{eV}$ and $\sim 9.1 \mathrm{eV}$ for an equivalent coincidence experiment energy of $\sim 10 \mathrm{eV}$ and $\sim 12 \mathrm{eV}$. The energy resolution of the apparatus was $1.2 \mathrm{eV}$. The energy of the electron beam was calibrated at low beam currents $(\sim 100 n A)$ by detecting the $19.3 \mathrm{eV}$ elastic resonance in helium [23]. The super-elastic data were taken at significantly higher beam currents $(\sim 6 \mu A)$, which resulted in an additional uncertainty in the energy of the experiment due to surface charging of calcium deposition on elements inside the vacuum chamber (this has been observed for calcium previously [6]). The effect of these deposits are difficult to estimate, but were expected to lead to an overall uncertainty in the electron energy of $\sim 2 \mathrm{eV}$.

The electron detector is of a standard hemispherical design and uses a 3-element zoom lens to image electrons from the interaction region onto the entrance of the energy analyser. The residual energy of the analyser was set $2.93 \mathrm{eV}$ above that of the gun to detect electrons superelastically scattered from the interaction region. The electron detector was moved around the interaction region so as to measure the super-elastic signal at different scattering angles $\theta_{e}$. This is a variation on the 'standard' super-elastic experiment which moves the electron gun rather than the detector. This alternative arrangement produces the same results as for the conventional measurements.

The calcium atomic beam was produced from a well collimated oven resulting in an atomic beam of narrow Doppler profile ( $70 \mathrm{MHz}$ FWHM) [24]. Atoms that passed through the interaction region were collected by a liquid nitrogen cold trap, so as to minimise deposition 
onto other surfaces inside the spectrometer [25]. The vacuum pressure inside the chamber was $\sim 2 \times 10^{-7}$ torr during operation.

The laser beam used in these studies was produced from a new system in the laboratory. This was a Spectra Physics Matisse Ti:Sapphire ring laser pumped by a 15W Millenia laser, and a Wavetrain external cavity frequency doubler which produced radiation at $\sim 423 \mathrm{~nm}$. The output power from the Wavetrain was set to $150 \mathrm{~mW}$ during operation, which is sufficient to excite more than $45 \%$ of the atoms in the interaction region to the $4{ }^{1} P_{1}$ state [23]. Fluorescence from this interaction was monitored, and the polarization of this radiation was found to be $>99.5 \%$, confirming that radiation trapping was not significant in these studies.

The Spectra Physics laser was far more stable in frequency compared to the Coherent MBR110 laser system used in previous work [5-7]. It was however still necessary to stabilise the laser for long periods of time, and this was accomplished by observing fluorescence from the interaction region inside the spectrometer so as to ensure the laser remained on resonance. At the energies used here the super-elastic yield could be very low (often $<1 \mathrm{~Hz}$ at higher scattering angles), and so it was necessary to continuously operate the experiment for several weeks to produce reliable data. The improved stability of the new Spectra Physics laser system ensured that this was possible.

A difficulty with low super-elastic yield was that the data accumulation time had to be long, and the background resulting from the elastic peak then subtracted. This was accomplished by invoking a computer controlled laser beam shutter into the experiment, so that the true signal could be derived. Accumulation times of several minutes were required for each data point at the higher scattering angles, resulting in several days operation to complete a set of data at a given angle. The results presented in this paper hence took around eight months to complete.

As noted previously [5-7] the technique adopted in Manchester does not measure the Stokes parameters, but rather determines the ACP's directly from measurement. This results in higher precision, in particular for the alignment angle $\gamma\left(\theta_{e}\right)$. To calculate $P_{l i n}\left(\theta_{e}\right)$ and $\gamma\left(\theta_{e}\right)$ the polarization vector $\hat{\mathbf{e}}$ of the incident laser beam was set linear by a Glan-laser polarizer, and was initially positioned parallel to the incident electron beam direction using a zero-order $\lambda / 2$ plate. The polarization vector was then rotated by an angle $\varepsilon$ through a total of $720^{\circ}$ in steps of $10^{\circ}$ by rotating the $\lambda / 2$ plate through $360^{\circ}$. A function of the form: 


$$
S_{\theta_{e}}(\varepsilon)=A_{\theta_{e}}+B_{\theta_{e}} \cos ^{2}\left(\varepsilon+\phi_{\theta_{e}}\right)
$$

was fitted to the data to establish $A_{\theta_{e}}, B_{\theta_{e}}$ and $\phi_{\theta_{e}} \cdot P_{l i n}\left(\theta_{e}\right)$ is then calculated using the expression:

$$
P_{\text {lin }}\left(\theta_{e}\right)=\frac{B_{\theta_{e}}}{2 A_{\theta_{e}}+B_{\theta_{e}}}
$$

$\gamma\left(\theta_{e}\right)$ is derived directly from the phase angle $\phi_{\theta_{e}}$ and scattering angle $\theta_{e}$ using the expression:

$$
\gamma\left(\theta_{e}\right)=\theta_{e}-\phi_{\theta_{e}}+n \pi
$$

where $n$ takes on integer values set by the geometry of the experiment (ie on which side of the detection plane the analyser is located). $\gamma\left(\theta_{e}\right)$ is further constrained to lie between $\pm \frac{\pi}{2}$ for direct comparison with theory.

To determine $L_{\perp}\left(\theta_{e}\right)$, circularly polarized incident radiation was required. A zero-order $\lambda / 4$ plate was hence inserted after the $\lambda / 2$ plate and the resulting laser radiation retro-reflected through the Glan-laser polarizer using a mirror. The $\lambda / 4$ plate was then rotated to minimise the transmitted signal, so as to ensure the correct orientation of the optic axis with respect to that of the $\lambda / 2$ plate. The $\lambda / 2$ plate was then rotated through multiple angles of $45^{\circ}$ to produce left- and right-hand circularly polarized radiation at the interaction region. The correct handedness of the radiation was determined by carrying out super-elastic experiments at $55 \mathrm{eV}$, where the sign of $L_{\perp}$ is well known [7]. $L_{\perp}\left(\theta_{e}\right)$ was then calculated using the expression:

$$
L_{\perp}\left(\theta_{e}\right)=\frac{S_{R H C}\left(\theta_{e}\right)-S_{L H C}\left(\theta_{e}\right)}{S_{R H C}\left(\theta_{e}\right)+S_{L H C}\left(\theta_{e}\right)}
$$

A final parameter $P_{t o t}\left(\theta_{e}\right)=\sqrt{P_{\text {lin }}^{2}\left(\theta_{e}\right)+L_{\perp}^{2}\left(\theta_{e}\right)}$ can be deduced that defines the degree of polarization, which is a measure of the coherence of the scattering process. For a fully coherent interaction $P_{t o t}=1$. 


\subsection{Experimental results and comparison to theory.}

\subsubsection{2eV equivalent energy.}

Figure 3 shows the experimental results for the higher incident energy with $E_{\text {inc }}^{c} \sim 12 \mathrm{eV}$, where the Stokes parameters $\left(P_{1}, P_{2}\right)$ have been calculated from $P_{\text {lin }}$ and $\gamma$ using equation 1. Since $P_{3}=-L_{\perp}$, only $L_{\perp}$ is shown. $P_{\text {tot }}$ is also depicted, calculated from $P_{l i n}$ and $L_{\perp}$.

The results for $P_{\text {lin }}$ (figure 3a) indicate that minima exist at angles $\theta_{e} \sim 65^{\circ}, 100^{\circ}$ and $125^{\circ}$, the minimum at the lower scattering angle being deepest at around 0.27 . The largest value of $P_{\text {lin }}$ is $\sim 0.93$ at $\theta_{e} \sim 115^{\circ}$, indicating a high degree of alignment at this angle. The alignment angle $\gamma$ (figure $3 \mathrm{~b}$ ) only slowly changes from $\gamma \sim-75^{\circ}$ at the lower scattering angles from $\theta_{e}=30^{\circ}$ to $\theta_{e}=85^{\circ}$ with a small dip to $\gamma= \pm 90^{\circ}$ at $\theta_{e}=60^{\circ}$, indicating that the charge cloud is aligned orthogonal to the electron beam direction at this scattering angle. The charge cloud reverses direction from this point, $\gamma$ increasing to $\gamma \sim-70^{\circ}$ at $\theta_{e}=70^{\circ}$, at which point it reverses direction again and once more decreases. The charge cloud is then observed to continuously rotate around the detection plane as the scattering angle increases from $\theta_{e}=70^{\circ}$ (note that results at $\gamma= \pm \pi / 2$ are equivalent). The charge cloud angle becomes relatively static at $\gamma \sim-55^{\circ}$ for scattering angles $130^{\circ} \leq \theta_{e} \leq 145^{\circ}$.

The parameter $L_{\perp}$ (figure $3 \mathrm{e}$ ) is of a relatively simpler form, and shows only a single large dip at $\theta_{e} \sim 105^{\circ}$, where $L_{\perp} \sim-1$. A similar magnitude occurs at $\theta_{e} \sim 60^{\circ}-70^{\circ}$, where $L_{\perp} \sim+1$. In both cases, the charge cloud is fully oriented, with the bound electrons orbiting in opposite directions. The parameter $P_{t o t}$ (figure 3f) lies mostly around unity, indicating that the interaction is fully coherent. There is a small decrease in this parameter at the highest scattering angles, which indicates a small loss of coherence in the interaction.

The derived Stokes parameters $\left(P_{1}, P_{2}\right)$ using equation (1) are shown in figures $3 \mathrm{c}$ and $3 \mathrm{~d} . P_{1}$ displays two main features at $\theta_{e} \sim 70^{\circ}, 110^{\circ}$ where local maxima are observed. At the higher scattering angle $P_{1} \sim 1$. By contrast, $P_{2}$ shows two broad maxima at $\theta_{e} \sim 60^{\circ}, 100^{\circ}$. Since the Stokes parameters do not directly relate to the charge cloud structure, they are less descriptive than $P_{\text {lin }}$ and $\gamma$. They are presented here as theoretical colleagues often only provide the 
calculated Stokes parameters. This is an historical artefact that has arisen from earlier coincidence studies, which measure the Stokes parameters rather than the ACP's. Since the super-elastic technique provides data of much higher precision than coincidence studies and can directly determine $P_{l i n}, \gamma$ and $L_{\perp}$, it would be useful if future theoretical results could also directly provide the ACP's from their calculations.

\subsubsection{Comparison between data at $12 \mathrm{eV} \& 10 \mathrm{eV}$.}

Figure 4 shows a comparison of the results at $\sim 12 \mathrm{eV}$ against those at $\sim 10 \mathrm{eV}$ for the parameters $P_{\text {lin }}, \gamma$ and $L_{\perp}$. The results at $10 \mathrm{eV}$ show a simpler structure than for $12 \mathrm{eV}$, although there are similarities between the data as might be expected. $P_{\text {lin }}$ at $10 \mathrm{eV}$ shows a shallower minimum in the forward direction than at $12 \mathrm{eV}$ for a scattering angle $\theta_{e} \sim 45^{\circ}$, with $P_{\text {lin }} \sim 0.55$. The dip at the higher scattering angle of $\theta_{e} \sim 130^{\circ}$ is of similar size to that at $12 \mathrm{eV}$. It is less clear if a dip exists at intermediate angles due to the poorer statistics at this energy. The observed minima in $P_{\text {lin }}$ at $10 \mathrm{eV}$ are broader than at $12 \mathrm{eV}$, and have moved apart.

The $\gamma$ parameter at $10 \mathrm{eV}$ also is seen to be relatively constant with $\gamma \sim-60^{\circ}$ at the lower scattering angles. The charge cloud angle does not however show the rapid change observed at $12 \mathrm{eV}$ when $\theta_{e} \sim 100^{\circ}$, but rather increases to $\gamma \sim 0^{\circ}$ at $\theta_{e} \sim 110^{\circ}$, after which it too reduces in angle as $\theta_{e}$ increases. There is evidence of a small dip around $\theta_{e}=65^{\circ}$ at $10 \mathrm{eV}$, but there are not enough data points to make a definite conclusion about this feature.

$L_{\perp}$ shows a simpler structure at $10 \mathrm{eV}$ (figure 4(f)) compared to $12 \mathrm{eV}$ (figure 4(e)), with a broad feature appearing at $\theta_{e} \sim 85^{\circ}$. The range of $L_{\perp}$ is reduced from +0.80 at $\theta_{e} \sim 125^{\circ}$ to 0.53 at $\theta_{e} \sim 85^{\circ}$ for this energy. The width of the minimum is larger at $10 \mathrm{eV}$ than at $12 \mathrm{eV}$.

Overall the results for the ACP's at the two energies are similar, but reflect the significant changes that can occur as a function of a small change in the incident beam energy. The results contrast significantly to those obtained at higher energies as in [6], which tend to show additional structures with correspondingly narrower features.

\subsubsection{Comparison between data at $10 \mathrm{eV}, 12 \mathrm{eV} \&$ theory.}

Figure 5 and figure 6 presents the data at $\sim 10 \mathrm{eV}$ and $\sim 12 \mathrm{eV}$ compared to the results from theoretical calculations at $10 \mathrm{eV}$. Comparison is made to both sets of experimental data, due to 
the uncertainty in the incident electron energy arising from contact potentials in the spectrometer for the high incident electron beam currents used here, as discussed in section 2. These potentials lead to an estimated uncertainty in the calibrated energy of $\sim 2 \mathrm{eV}$. The relative energy between the data sets should however be accurate at $\Delta E=2 \mathrm{eV}$, since the operating conditions of the spectrometer were the same at each energy.

Four different calculations are depicted, taken from data already published for the Stokes parameters. The models include a relativistic Distorted wave calculation (RDW) [19], an Rmatrix calculation (RM) [21], an R-matrix calculation using B-Splines (BSR) [22] and a convergent close coupling calculation (CCC) [20]. The R-matrix calculations use different numbers of states to converge ( 24 for the RM method, and 39 for the BSR method). Since the published data only provide Stokes parameters, the associated atomic collision parameters have been re-derived from the theory to allow comparison with experimental data, as detailed above.

An immediate observation from this comparison is that no theory agrees well with the experimental data for $\sim 10 \mathrm{eV}$, apart from in overall structure (figure 5). Each theory predicts different positions of the peaks and troughs, although there are similarities in the overall number of features predicted. As an example, the CCC, RM and BSR calculations all show peaks and troughs at similar scattering angles for $P_{1}, P_{2}$ and $L_{\perp}=-P_{3}$ (figures $5 \mathrm{c}, 5 \mathrm{~d}$ and $5 \mathrm{e}$ ) although their magnitude varies significantly between different models. The RDW calculation shows large differences compared to all other calculations, with the same number of features but with magnitudes and positions of the observed features quite different to other models.

The Stokes parameters $P_{1}, P_{2}, P_{3}=-L_{\perp}$ derived from experiment also show broadly similar structures to the calculations, but the position and magnitude of the features do not agree with any model at this energy. For $L_{\perp}=-P_{3}$ the RDW model is in closest agreement with the data, however for $P_{1}$ the RDW model predicts a minimum at $\theta_{e} \sim 70^{\circ}$ compared to a maximum in other calculations. $P_{1}$ is better represented by the other three models. No model predicts the data for $P_{2}$, apart from at scattering angles $\theta_{e}>105^{\circ}$ where agreement is reasonable. The $\mathrm{CCC}, \mathrm{BSR}$ and RM models do not reproduce the position of the minimum for $L_{\perp}$, and overestimate the magnitude of this parameter at the lower scattering angles. 
Since the Stokes parameters (figures (c) - (e)) are not in good agreement with experiment, the ACP's derived from each model are also not in good agreement, as seen for $P_{l i n}$ and $\gamma$ (figures 5a, 5b). For $P_{\text {lin }}$ no model agrees well with the data. For the $\gamma$ parameter the CCC and BSR models predict a local minimum at $\theta_{e} \sim 50^{\circ}-60^{\circ}$ but both predict this minimum to be at $\gamma \sim \pm 90^{\circ}$, in contrast to the data which finds $\gamma \sim-60^{\circ}$ over a wide range of scattering angles. Both models predict the charge cloud to reverse direction at $\theta_{e} \sim 65^{\circ}-70^{\circ}$ which is not observed. The RM model predicts a minimum at $\theta_{e} \sim 68^{\circ}$ with $\gamma \sim+9^{\circ}$, before reversing direction at $\theta_{e} \sim 80^{\circ}$, in complete disagreement with the data. The RDW model is in poorest agreement with the data overall, apart from at the higher scattering angles with $\theta_{e}>105^{\circ}$ where all models predict the results reasonably well.

The data indicate that there is some loss of coherence in the interaction, as given by $P_{t o t}$ (figure 5f). In particular there is an observed reduction from $P_{t o t}=1$ at $\theta_{e} \sim 45^{\circ}$ that is predicted by the RDW calculation. The calculated loss of coherence observed at the higher angles is not predicted.

Given the poor agreement between theory and experiment for the data at $\sim 10 \mathrm{eV}$, and since the absolute energy of the experiment has an uncertainty $\sim 2 \mathrm{eV}$, the results for $12 \mathrm{eV}$ are also plotted against theory in figure 6. There is now far better agreement between the data and the predictions. The RDW model still does not agree with experiment. The R-Matrix model is in much better agreement, apart from for $\gamma$ where it deviated strongly from the data at the lower scattering angles. The RM model predicts $P_{\text {lin }}$ and $L_{\perp}$ well, although the minimum in $P_{\text {lin }}$ at lower scattering angles is wider and deeper than the data.

The most accurate models are the R-matrix using B-Splines (BSR) and the convergent Close Coupling theories (CCC). The BSR does not obtain the correct position for the dips in $P_{\text {lin }}$ or $L_{\perp}$, however the magnitudes of these features are well produced. The results for $\gamma$ are in good agreement with the data, but once again there appears to be an angular shift in the features compared to experiment. The CCC model accurately predicts the positions of all features in the data, and is reasonably accurate in predicting the magnitudes. In particular, the agreement between the $\mathrm{CCC}$ model and data for $\gamma$ is particularly impressive, with all features accurately reproduced. This model appears to give the closest overall prediction of the data. 
It appears from the close agreement between the calculations at $10 \mathrm{eV}$ and the data taken at $12 \mathrm{eV}$, that there is an offset in the absolute value of the experimental energy of $\sim 2 \mathrm{eV}$. This is almost certainly due to charging of calcium deposits on surfaces inside the spectrometer. This is an effect that has been observed previously for calcium [5-7], however it plays a more critical role in these experiments at low energies due to the higher beam currents that are required to produce statistically meaningful data.

It is worth noting that since the overall energy resolution of the experiment is $\sim 1.2 \mathrm{eV}$, the most accurate comparison with theory requires the calculations to integrate their results over this energy resolution. This is particularly important when the parameters change significantly with energy, as is observed here. It is unlikely that resonance are significant at these energies, however all calculations need to carefully consider the effects of this energy resolution particularly at these low energies, to provide a robust comparison with the experimental results.

\subsection{Conclusion \& Future Work.}

In this study we have presented new experimental data for electron impact excitation of calcium at low energies, measured using the super-elastic scattering technique. Data have been taken for $P_{\text {lin }}, \gamma$ and $L_{\perp}$ at equivalent incident energies of $\sim 10 \mathrm{eV}$ and $\sim 12 \mathrm{eV}$. The results have been compared to calculations using R-matrix, CCC and relativistic distorted wave models. It has been found that an offset of $\sim 2 \mathrm{eV}$ in the data is likely, since the results at $\sim 12 \mathrm{eV}$ agree well with close coupling theories for $10 \mathrm{eV}$, whereas there is little agreement with the data at the lower energy. It will be interesting to see if calculations performed at $8 \mathrm{eV}$ agree with the data at the lower energy presented here. For a proper comparison to be made, all models will need to carefully consider the energy resolution of the spectrometer, since the derived parameters are seen to vary significantly as a function of this energy.

The super-elastic method is the only technique that can provide high precision data over a wide range of scattering angles for comparison to theory. It is therefore important to develop this technique for use with other targets, in particular those which have importance in practical applications such as in plasma research and lighting. The super-elastic technique is however limited to targets that can be excited by existing laser radiation. Recent innovations in high resolution lasers has allowed a much wider range of wavelengths to be produced, and so the opportunity now arises to explore these interactions for many other targets. This will 
provide new and precise data to further refine the models describing the scattering process, with a goal to establishing a universal model that will predict electron excitation of all targets.

\section{Acknowledgements}

The Engineering and Physical Science Research Council is acknowledged for providing funding to carry out this research, for providing part of a studentship to SJ, and for providing a postdoctoral research position for $\mathrm{MH}$.

\section{References.}

[1] Andersen N, Gallagher J W \& Hertel I V (1988) Phys. Rep. 1651

[2] Andersen N and Bartschat K (1996) Adv. At. Mol. Phys. 36, 1

[3] Hertel I V and Stoll W (1974) J. Phys. B 7 570; J. Phys. B 7583

[4] Hertel I V and Stoll W (1977) Adv. At. Mol. Phys. 13113

[5] Hussey M, Murray A J, MacGillivray W R and King G C (2007) Phys Rev Lett 99133202

[6] Murray A J and Cvejanovic D (2003) J Phys B 364875

[7] Hussey M, Murray A J, MacGillivray W R and King G C (2008) J Phys B 41055202

[8] Hall B V, Shen Y, Murray A J, Standage M C, MacGillivray W R and Bray I (2004) J Phys B 371113

[9] Farrell P, MacGillivray W R and Standage M C (1988) Phys. Rev. A 374240

[10] Stockman K A, Karaganov V, Bray I and Teubner P J O (1998) J. Phys. B 31 L867

[11] Karaganov V, Bray I and Teubner P J O (1999) Phys Rev A 594407

[12] Slaughter D S, Karaganov V, Brunger M J, Teubner P J O and Bray I (2007) Phys Rev A 75062717

[13] Zetner P W, Johnson P V, Li Y, Csanak G, Clark R E H and Abdallah J (2001) J Phys B 341619

[14] Johnson P V, Eves B, Zetner P W, Fursa D and Bray I (1999) Phys Rev A 59439

[15] Scholten R E, Karaganov V, Teubner P J O and Farrell P M (1999) Phys Rev A 60330

[16] Farrell P M, MacGillivray W R and Standage M C (1991) Phys Rev A 441828

[17] Meng X K, MacGillivray W R and Standage M C (1992) Phys Rev A 443162

[18] Murray A J, MacGillivray W R and Hussey M J (2008) Phys Rev A 77013409

[19] Chauhan R K, Srivastava R and Stauffer A D 2005 J. Phys. B 382385

[20] Fursa D V and Bray I (2008) J Phys B 41145206

[21] Kawazoe S, Kai T, Chauhan R K, Srivastava R and Nakazaki S (2006) J Phys B 39493

[22] Zatsarinny O, Bartschat K, Bandurina L and Gedeon S (2007) J Phys B 404023

[23] Brunt J N H, King G C, and Read F H (1977) J. Phys. B 101289

[24] Murray A J, Hussey M J and Needham M (2006) Meas Sci Tech 173094

[25] Murray A J and Atkinson S(2004) Meas Sci Tech 15 N31 
Figures.

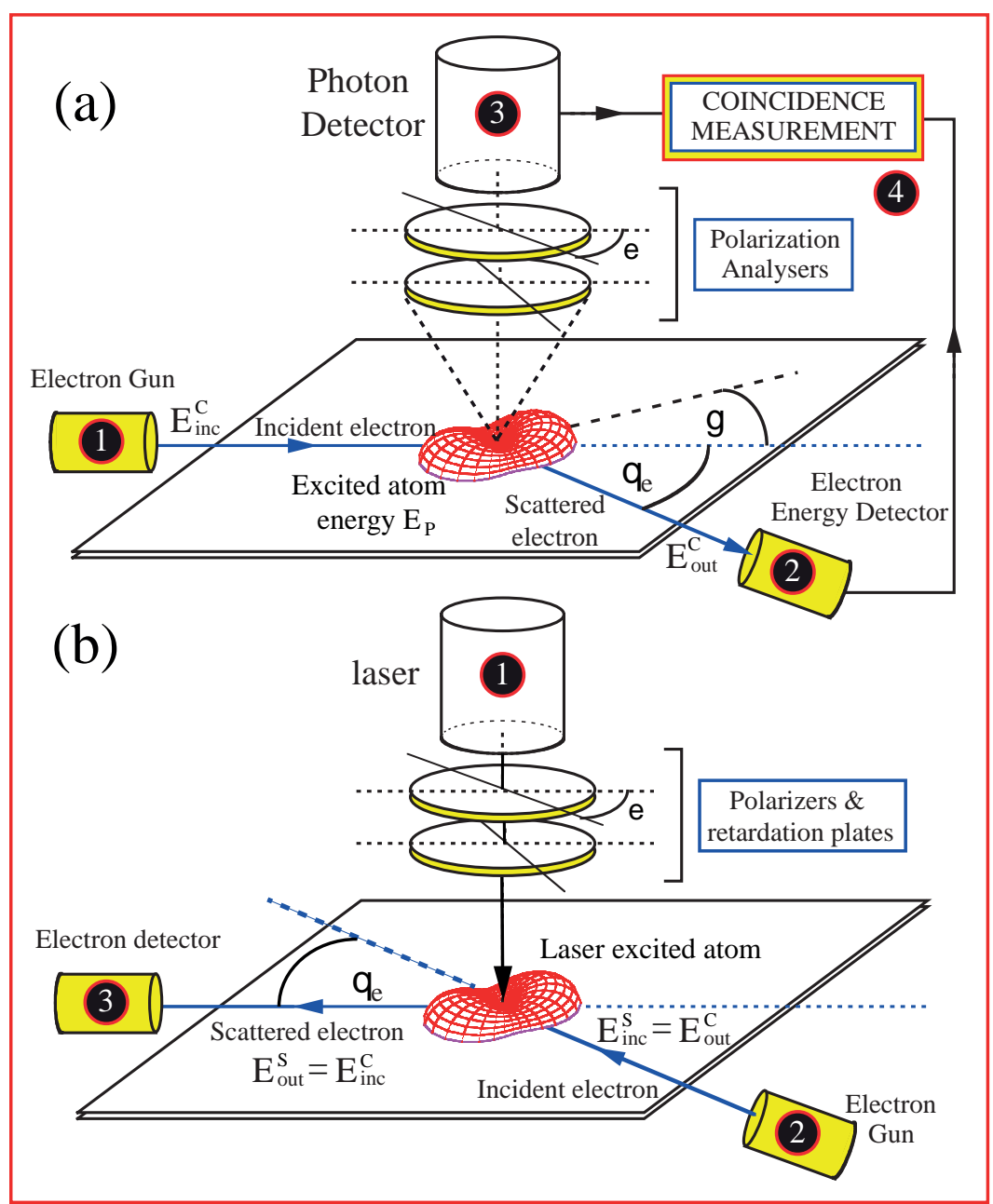

Figure 1. (a).The coincidence scattering geometry, which requires 4 steps to ascertain the collision parameters $P_{l i n}, \gamma, L_{\perp}$. (b) The super-elastic geometry, which requires 3 steps. For details see text. 


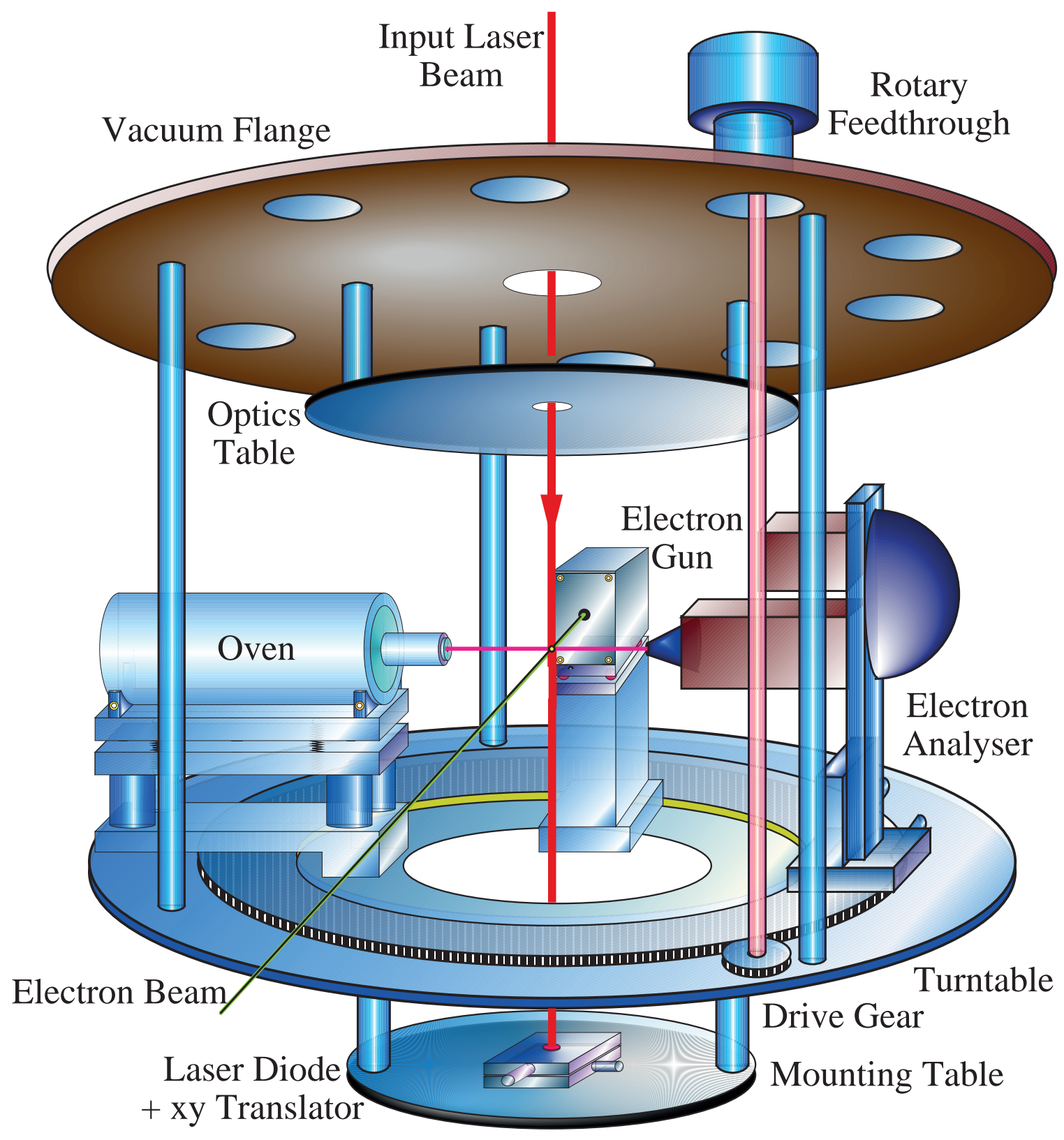

Figure 2. The super-elastic spectrometer, showing the electron gun, analyser and oven. The laser beam enters through a window on the top flange, and is directed orthogonal to the scattering plane, as set by a tracer laser diode inside the spectrometer. 


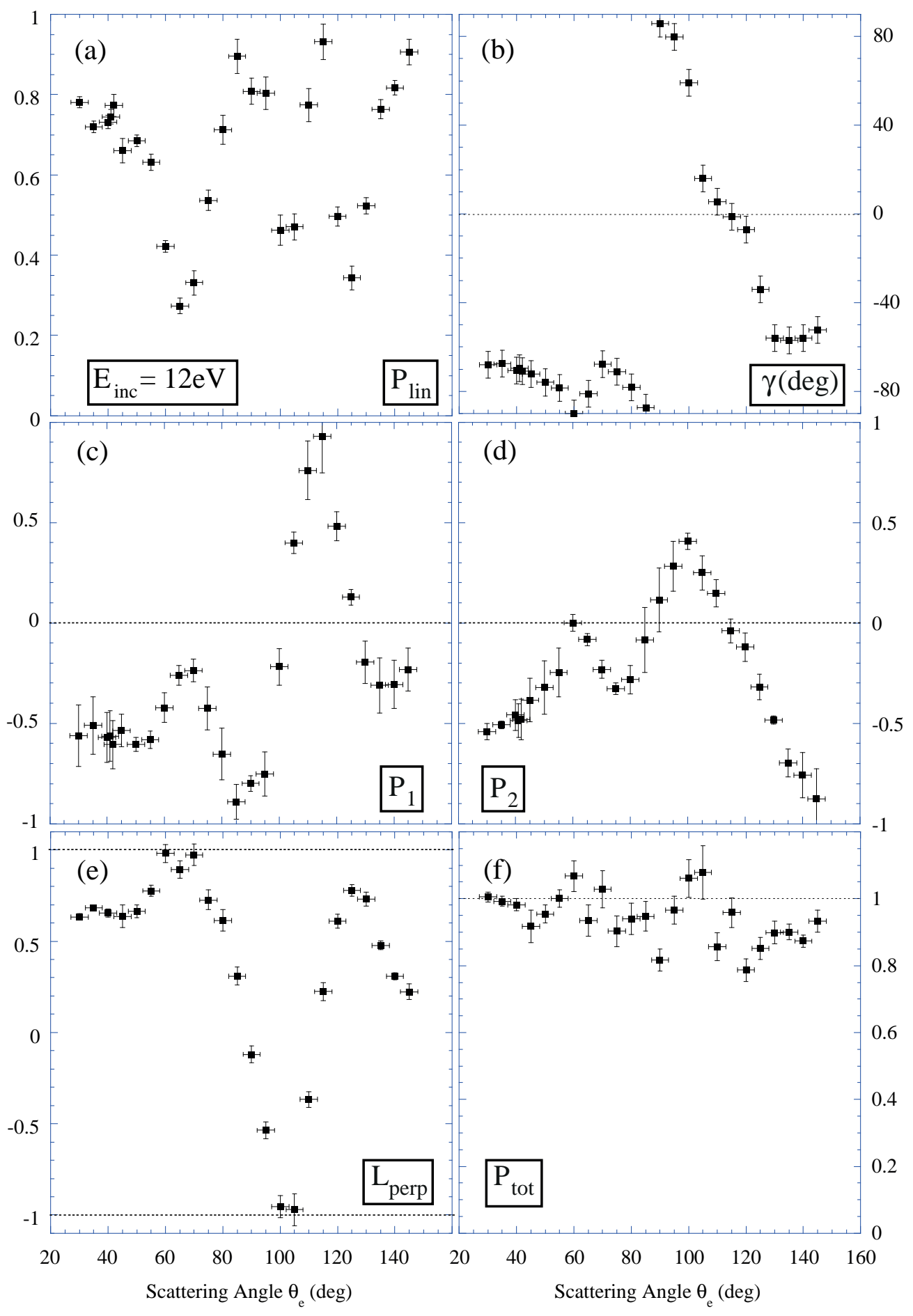

Figure 3. Data at $12 \mathrm{eV}$, showing the ACP's, the derived Stokes parameters and the coherence parameter $\mathrm{P}_{\text {tot. }}$. 


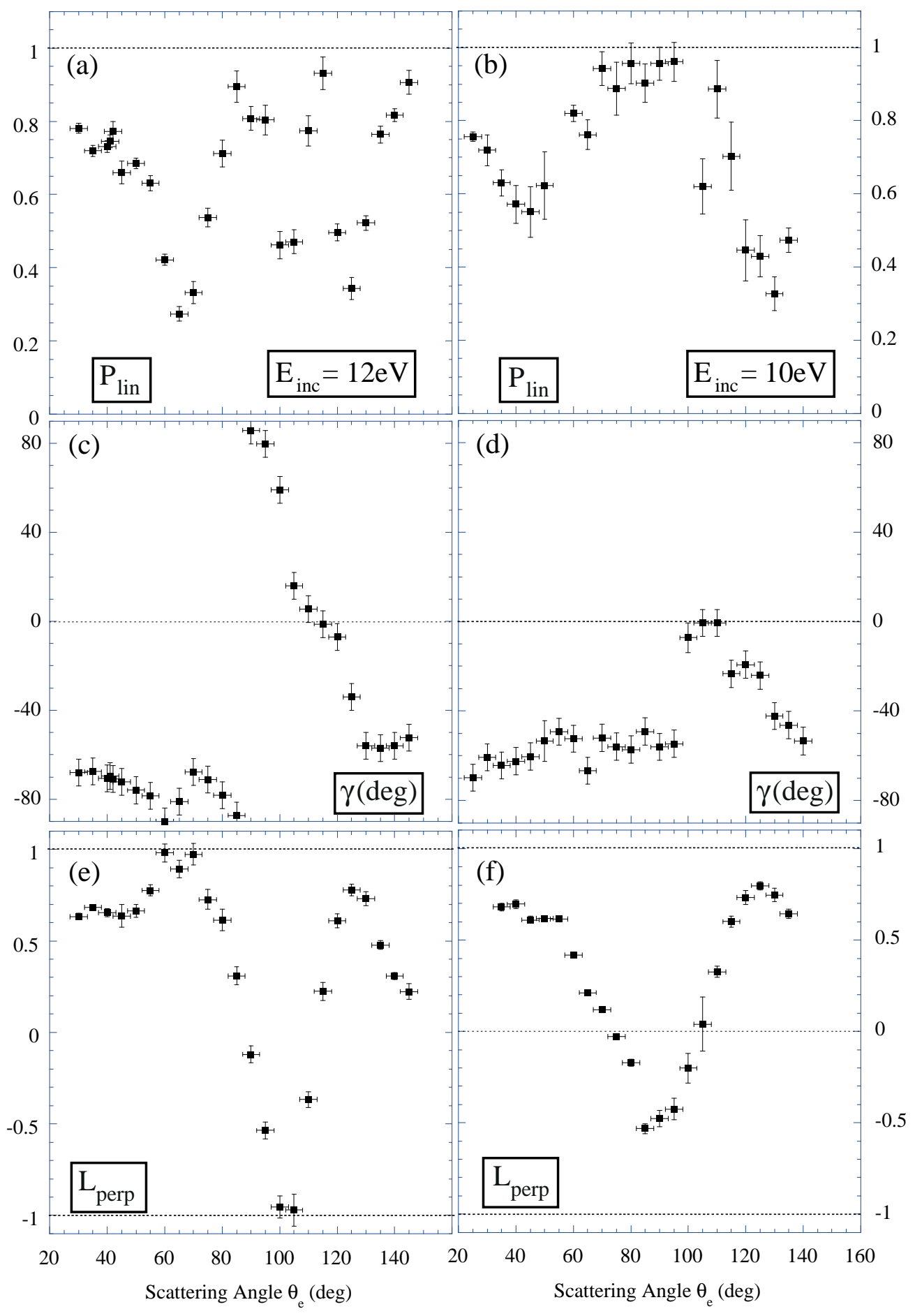

Figure 4. Comparison between the measured ACP's at $12 \mathrm{eV}$ and $10 \mathrm{eV}$. 


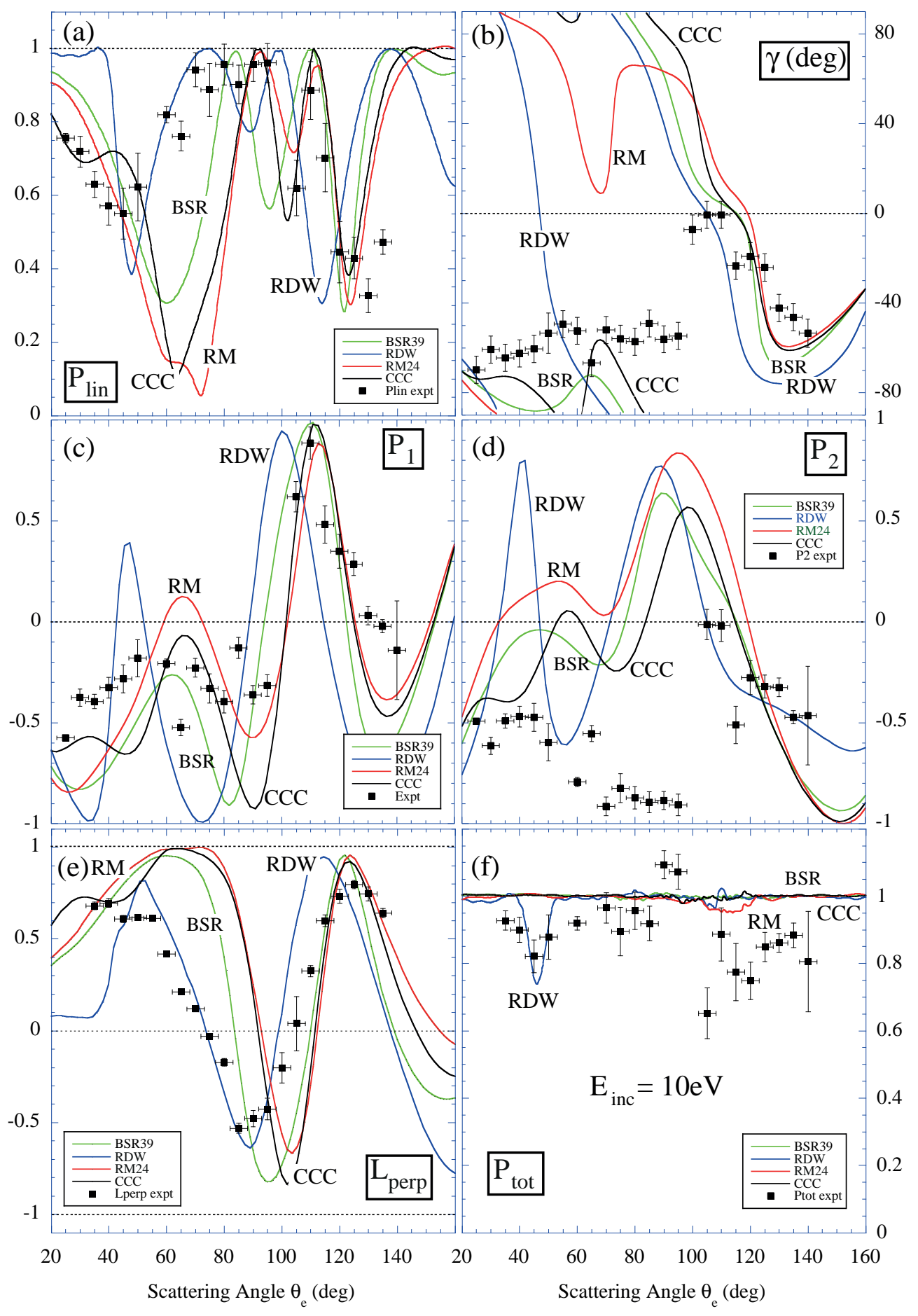

Figure 5. Data at $10 \mathrm{eV}$ nominal energy plotted against different theoretical models calculated for an incident energy of $10 \mathrm{eV}$. Poor agreement is found between theory and experiment. For details see text. 


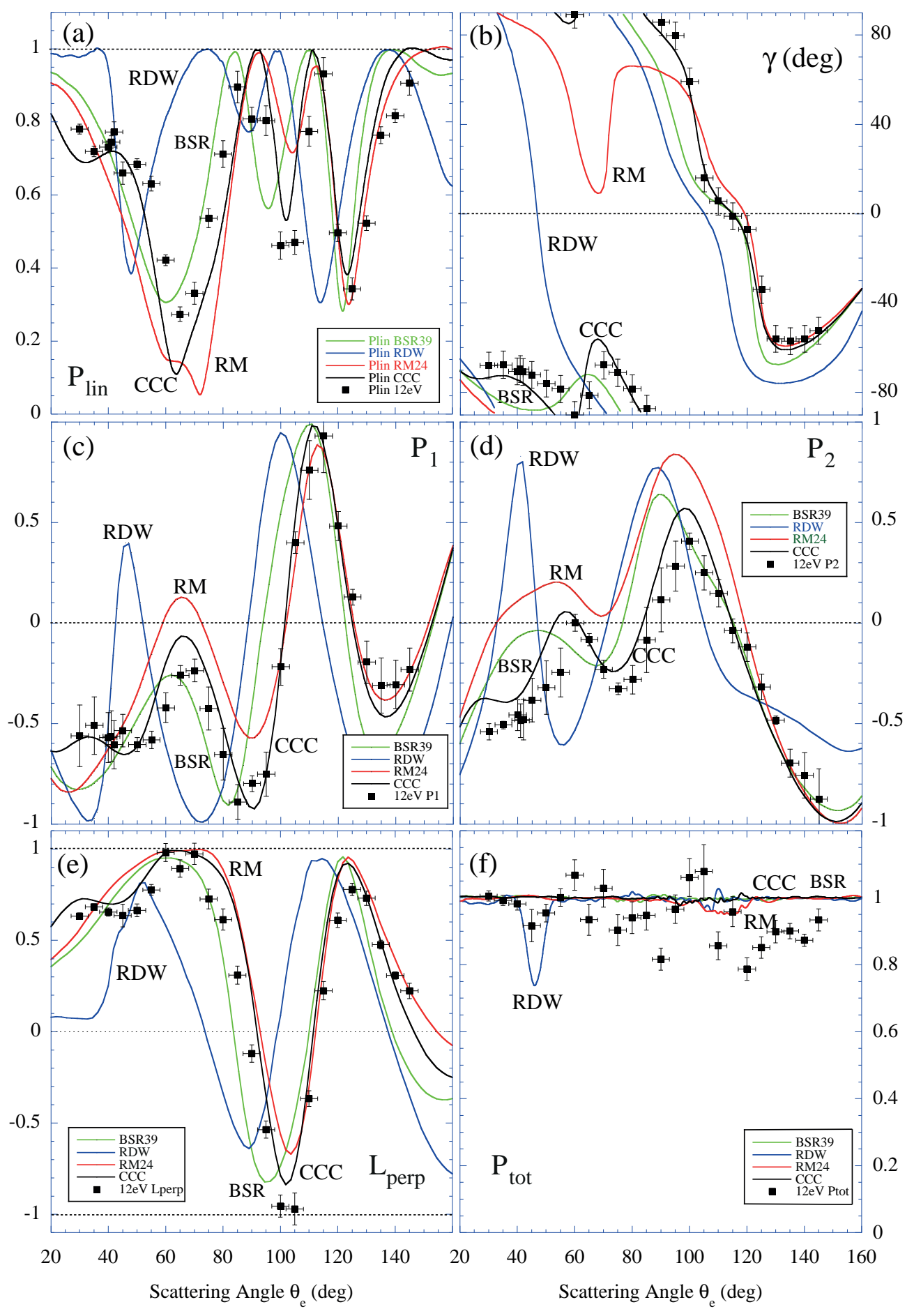

Figure 6. Data at $12 \mathrm{eV}$ nominal energy plotted against different theoretical models calculated for an incident energy of $10 \mathrm{eV}$. Good agreement is found between the close coupling theories and the data at this energy. 